\title{
Current and future role of ultrasound in the emergency department
}

Department of Radiology, Hope Hospital, Manchester H C Burnett D A Nicholson

Correspondence to: Dr David A Nicholson, Consultant, Directorate of Clinical Radiology, Hope Hospital, Stott Lane, Salford M6 8HD.

Accepted 31 October 1998

\author{
Hugh C Burnett, David A Nicholson
}

The previous article by Robinson and Clancy examined the value of ultrasound in the international perspective, placing the role of emergency department ultrasound in this country in the context of practice elsewhere in the world.

This article seeks to explore the role of ultrasound in casualty-where it fits and where its limitations lie in relation to the advantages of alternative imaging modalities. The "who" of imaging will also be addressed-who should be performing ultrasound and who should train them. Anticipated future trends will be explored.

\section{Relevance of ultrasound}

The role of imaging within the emergency department has changed substantially. Patients and clinicians now expect imaging questions to be addressed immediately. The sophistication of the new imaging techniques secures diagnoses and allows early and appropriate management. In the application of ultrasound in casualty there has been extensive exploration in the literature of two fields: first the early, instant assessment of the trauma victim and second the role of ultrasound in the general daily case load of the emergency department.

In most centres imaging is currently performed on a discipline dictated basis. Thus when an obstetric question arises this is commonly imaged in an ultrasound department by radiographers on dedicated obstetric lists or by obstetricians. When a medical question arises, such as the possibility of deep venous thrombosis, a referral to a radiologist's ultrasound list is made. After referral the patient is transferred to the appropriate imaging department. This is a system convenient to the hospital staff that run the system rather than the patient.

Ultrasound is a modality well suited to the casualty department. For the imaging facility to visit the patient is a neat solution attractive to the patient. Were there a machine "just next door" it seems logical that it would be used often and find application to a multitude of questions.

All imaging modalities have evolved with great rapidity in the last decade. There is merit therefore in making a regular and conscious attempt to determine if current medical practise lies at odds with the potential and limitations of the imaging modalities that support it.

\section{Competing modalities} COMPUTED TOMOGRAPHY

There is a trend for computed tomography scanners to be sited close to or within the casualty department, particularly in centres with a large trauma workload. At the very time that ultrasound has risen as a modality offering ease of availability, the availability of its alternative also rises. Models supporting the value of ultrasound that assume a time, transport, and availability cost for computed tomography are already part outdated. Ultrasound must stand on the quality of the information it offers not just the rapidity of its availability.

The quality of information provided by computed tomography has transformed radiological decision making. Spiral scanning with subsecond rotation times and high heat capacity tubes have enormously accelerated the speed of acquisition. Very high quality images in the current generation of scanners have increased accuracy of diagnosis. They routinely detail subtleties over a wide range of applications meaning that quoted pick up rates for other modalities, including ultrasound, have meaning only in articles comparing the latest equipment.

Computed tomography has consistently higher sensitivity for parenchymal injuries in trauma; it also outperforms ultrasound for questions regarding intestinal pathology and injury. It performs with excellence in areas that ultrasound is weak, for example in imaging bony and gas containing structures (lung and bowel). If computed tomography is very easily available and can give answers of great accuracy-superior to ultrasound-then this raises the question whether ultrasound is left the role only of a method of triage, determining which patient requires instant access to theatre and which is stable enough for computed tomography. To say that time and availability restrictions would impede access to computed tomography is an argument of rationing rather than of technical merit.

\section{PLAIN RADIOGRAPHS}

Plain radiographs are also rapidly and routinely available in the emergency department setting and retain a defined role. Studies comparing the utility of ultrasound detection of pleural fluid with chest radiography have identified no performance advantage. ${ }^{1}$ With radiography likely to remain routinely available, the addition of a test without added yield should be questioned.

It is useful to consider in a little depth some of the quoted common indications for casualty based ultrasound.

\section{(1) Abdominal pain}

Consider abdominal pain, which accounts for up to $18 \%$ of casualty attendances. ${ }^{2}$ It is not, of course, simply the technology or patient demographics that shift. The gold standard for 
medical care also shifts. The management of acute pancreatitis is one example. The traditional practice of a raised serum amylase directing a conservative management line to drip and suck is challenged by current evidence, directing that very early diagnosis of underlying biliary calculus disease mandates urgent or emergency endoscopic retrograde cholangiopancreatographic examination with a view to sphincterotomy.

Arguably then all patients presenting with abdominal pain should be screened for the presence of gall stones. Current practice dictates that casualty assessment leads to referral to a medical or surgical team depending on the overtone of the patient's history. Subsequent referral to an imaging department at third hand is delayed and dependent on it being open and receptive. This screening could be performed in casualty before referral and could save hours and potentially lives.

There is a certain role for ultrasound in the depiction of gall stones and as an aid to the diagnosis of acute cholecystitis and acute appendicitis. It should be appreciated that a high quality examination seeks not only accurate diagnosis but the accurate detection of the array of associated complications. For example in gall stone disease accurate detection of cystic duct stones, common bile duct stones, and perforation should be expected. Realistically this takes hours of training and considerable case exposure. This will only come with a concentration of case experience, and there is a strong case that this would be absent if a broad range of staff in any department were each seeing few cases.

Of course it is an option to perform a quick examination that will successfully and usefully demonstrate normality in many patients, but patients with positive diagnoses should realistically expect and will increasingly demand quality.

\section{(2) Deep venous imaging}

The appropriate test and the service provision of deep venous imaging is an area extensively explored in the radiological literature. ${ }^{3}$ Important issues of quality assurance bear mention.

The recognition of a positive test for thrombus using grey scale ultrasound in a nonoedematous leg is a relatively straightforward skill easily taught. The specificity of a positive diagnosis is high. Base venous imaging in casualty and such positive cases would receive appropriate treatment earlier only if patients are not routinely heparinised on the suspicion of thrombus.

But patient attendance in casualty will only be shortened if a confident negative diagnosis can be obtained. Normality, particularly in the swollen, large, or oedematous leg, is a far harder condition to establish even with confident colour Doppler imaging skills, especially as is common medical consultants would anticoagulate below knee thrombus. In these cases a full and dedicated colour or power Doppler examination well performed on the best, and not bedside, equipment can take 20 minutes. There is a considerable miss rate or false nega- tive rate even on high quality powerful machinery in a dedicated darkened suite.

The argument here is while it is relatively easy to confirm the diagnosis, it is harder to rule it out. Thus positioning the imaging test in casualty while expediting management in the positive case may not save admission safely if negative and thus may not be cost saving. The outcome after introduction of a new test thus may lie at odds with preintroduction expectations.

\section{(3) Obstetric and gynaecological ultrasound}

All radiologists gain some experience in obstetric ultrasound. Enough to realise that mastery is a field different and beyond that of routine abdominal scanning. Very few would claim competence.

Their state of training is thus in some ways similar to that of casualty staff. Large series have examined the sensitivity and reasonable specificity of detection of several simple base parameters. ${ }^{45}$ These include the presence of free pelvic fluid and the beating fetal heart. Within these parameters reasonable figures have been attained. But it should be appreciated that these are limited goals. To sit with specifically skilled radiographic staff in their pursuit for an example of a partial placental abruption is an education. If the gold standard remains a scan by such staff then a more limited casualty assessment can fit only as part of clearly drawn up interdepartmental protocols to ensure that patients who require dedicated and detailed scans are not missed.

Other series have included endovaginal ultrasound as part of the routine protocol in the assessment of possible ectopic pregnancy. ${ }^{6}$ This is a difficult, invasive, and specialist skill that many clinicians would consider appropriate after specialist consultation rather than forming part of routine work up.

While few emergency departments offer this service it highlights a fundamental choice. Casualty based ultrasound can be a triage tool-operating on simple parameters offered by many staff or it can offer a service requiring intense training and offering high level answers. It should be appreciated that if the first option is taken the place of specialist ultrasound remains.

\section{Trauma}

Ultrasound in the trauma setting has a different array of attractions and demerits, and there is very extensive literature on the application of ultrasound..$^{7-10}$

In the setting of acute trauma considered judgment is not an available luxury. The premium is for a rapid opinion that is right first time. There is little or no room for equivocation.

(1) EVOLUTION OF THE ROLE OF ULTRASOUND IN TRAUMA

Recent literature on trauma ultrasound has focused on the detection of free fluid. Diagnostic peritoneal lavage has previously held the forthright role of a test with high sensitivity for 
intraperitoneal blood serving as a proxy marker for organ damage. It is fast to perform and has high sensitivity. It is, however, non-specific and provides no primary information on parenchymal injury; all is inferred. It requires staff capable of interpreting the test rapidly. Many trauma protocols dictate that a patient be observed clinically for 24 hours as an inpatient on a negative result on diagnostic peritoneal lavage if no additional diagnostic test is performed.

Diagnostic peritoneal lavage has shaped the pattern of introduction of ultrasound certainly in the United States. Ultrasound has been assessed and applied on its capability to detect free fluid. Thus free fluid has been taken as the first proxy marker of organ injury for both techniques. In this comparison ultrasound compares well and can reliably and rapidly demonstrate fluid. Surgeons have demonstrated such sensitivity to pathology on ultrasound scanning on the basis of quite simple and limited training. For example in one trauma teaching programme imaging was based on ultrasound probes being placed in four standard locations (the right and left upper quadrants, the epigastrium, and the pouch of Douglas). Such standardised imaging protocols, however, do not make full use the inherent flexibility of ultrasound.

\section{(2) FULL POTENTIAL OF ULTRASOUND}

Ultrasound has a long history of application to the depiction of solid abdominal organ pathology and anatomy. To restrict its application only to the detection of free fluid is to underestimate its potential application. However localised injury to solid organs has a variable presentation on ultrasound. Focal parenchymal lacerations may appear anechoic, hypoechoic, or hyperechoic and show an alteration in normal anatomy.

While ultrasound has a clearly demonstrated inferior sensitivity to major organ injury when compared with computed tomography, it has a proven ability to detect major hepatic splenic and renal injuries and thus instantly select patients that require theatre.

Ultrasound has unique advantages:

(A) It can be performed immediately, simultaneously, and in parallel with clinical management. This can hasten patient triage and has been demonstrated to have beneficial application in selection of cases requiring laparotomy with a reduction in the time spent in computed tomography.

(B) It can be performed on the unstable patient and requires no relocation or disturbance of the patient.

(C) It can improve and focus the use of computed tomography.

In one large study from Miami the trauma application of ultrasound led to only a marginal decrease in the use of computed tomography. ${ }^{8}$ With prior ultrasound, however, computed tomography can be used with greater focus allowing optimisation of contrast enhancement to visualise a specific organ demonstrated to be abnormal on ultrasound.

\section{Key issues}

- Relevance of ultrasound

- Who should be performing ultrasound

- Direction of future change

It may also avoid computed tomography being performed as a blind screening examination in the patient lacking a reliable history due to neurological injury or intoxication. In these circumstances a normal ultrasound examination and stable physical examination and haemodynamic parameters may allow conservative management.

(D) Potential cost savings. This however is complex. The potential saving of radiology charges through surgeon performed ultrasound has been examined in the United States and has demonstrated a reduction in case management cost. ${ }^{11}$ The situation in most British departments is quite different with radiology departments operating on a bulk service contract rather than an individual case basis. Most hospitals would accept that the radiology department would expect to remain central in the training and quality supervision of casualty staff, and would probably remain as the final arbiter in difficult imaging questions. Importantly the radiology department would expect to remain closely involved in service provision in casualty, being uniquely placed to offer the range of available modalities and having insight into the qualities and appropriate place of each.

\section{Who should perform the trauma ultrasound?}

The bulk of the literature on the casualty application of ultrasound by surgical and emergency department staff has appeared outside the radiology literature.

In the United States ultrasound of trauma is performed largely by surgeons, which carries certain inherent advantages:

(A) The relevant staff are immediately available within the department when a trauma patient arrives.

(B) Staff with a knowledge of the patient's presentation and a trauma focus perform the ultrasound. Ultrasound produces images that are by nature subjective. If a surgeon is to operate on the basis of them there is an argument that he or she directly supervise those images.

There are however advantages in employing imaging specialists:

(A) Time in the trauma room is vital and should be managed with maximum efficiency. Tasks can be performed by staff serially or in parallel, with multiple tasks each performed by relevant personnel. The efficiently run trauma unit will utilise the second model with separate tasks (securing and managing the airway, securing vascular access, etc) appropriately delegated. To remove these staff and involve them with imaging would be acceptable only if there was not an alternative.

(B) There are successful models of trauma imaging by the on call radiology team, for 
example with on site resident specialist trainees with off site support from both imaging and interventional consultants. To be of relevance to the trauma team leader this radiology service must be promptly available and requires radiologist integration to the trauma team. By being within the trauma room the radiologist is in a position to supervise and give feedback on radiographic procedures and to direct additional imaging and tests.

The subjective nature of hard copy ultrasound images can be obviated if the radiologist demonstrates to the surgeon or team leader a relevant and focused summary of the important positive and negative findings in real time.

It must be accepted that if the radiology service is to provide a trauma ultrasound service then examination, interpretation, and clinical correlation should be immediately available around the clock in resuscitation areas. There are relatively few centres in the UK where the radiologist is integrated formally within the trauma team. It is probable that this will be available only in teaching centres with readily available junior staff or highly motivated on call consultants.

The costs of employing specifically skilled staff:

(A) A resident radiology service carries a heavy time burden and will have a low efficiency particularly if the trauma load is light. Twenty four hour availability can be expected to disrupt daytime schedules in the radiology department the next day.

(B) It is argued that their performance and interpretation will be more timely if performed by the clinical team. ${ }^{4}$ But this is not necessarily the case as there are many examples of practice directly incorporating the radiologist within the trauma team. Of course, the time and disruption to routine schedules must be accepted by the radiology service if it is to make this commitment, allowing proper integration into the trauma service.

\section{Credentialising issues in ultrasound}

Two quite different positions regarding training have been adopted with regard to ultrasound. On the one hand, particularly in the United States, local courses (including one day workshops to familiarise surgeons with ultrasound physics and to observe videotape of practical ultrasound) provide an introduction and allow juniors to begin imaging. ${ }^{82}{ }^{13} \mathrm{~A}$ figure of around 50 trauma ultrasounds providing a plateau in terms of competence is quoted. ${ }^{14} 15$

The alternative position is that taken by the Royal College of Radiologists (RCR) in the UK, which has laid down centralised and

Table 1 Advanced ultrasound capabilities

\begin{tabular}{|c|c|}
\hline Feature & Advantage \\
\hline \multicolumn{2}{|l|}{ Doppler facilities } \\
\hline$M$ mode & $\begin{array}{l}\text { Allow accurate measurement of blood flow velocity and allow assessment } \\
\text { of the resistance of an arterial bed }\end{array}$ \\
\hline Colour & Detailed depiction of blood flow aiding venous imaging \\
\hline Power & $\begin{array}{l}\text { Sensitive to low volumes of vascular flow, allowing assessment of the } \\
\text { volume of flowing blood, without directional information }\end{array}$ \\
\hline Harmonic imaging & $\begin{array}{l}\text { Offer considerably improved spatial and contrast resolution over } \\
\text { conventional ultrasound }\end{array}$ \\
\hline
\end{tabular}

demanding guidelines with a view to them being adopted as national guidelines. ${ }^{16}$ Because the casualty application of ultrasound in the UK has lagged behind the United States, the RCR has been in a position to react to the United States experience, which gives advantages in terms of ensuring levels of quality and centralising acceptable standards.

The laid down standards are considerably tougher than those described in local centres in the United States, where some claim that junior surgical trainees with a service record of less than 15 ultrasounds and with "minimal training" could perform ultrasound at a level comparable with that of attending radiologists. The RCR has directed that 300 cases annually be undertaken to maintain competence. This sort of case load is likely to be performed only if selected senior staff in the emergency department specialised and controlled the imaging facility.

All experienced in ultrasound imaging recognise how easy it is to miss significant pathology. Given very limited objectives (for example the detection of free fluid only) ultrasound can be taught easily to staff with limited imaging experience. However this is to deliberately forego much of the potentially available information. This potential information missed should be considered in terms of implications on patient outcome, acceptability to the patient, and balanced against the costs of employing specifically skilled staff.

Medicolegal issues in ultrasound imaging Ultrasound is by essence a real time modality. Many papers based on a live surgical interpretation compare retrospective review of hard copy images by senior radiologists. They find interpretation similar. But the essential question raised is-what would that experienced imaging specialist have seen live? What images would he or she have taken? In computed tomography there is some case guided improvisation in technique by the experienced operator, but the end product images are by nature more objective.

\section{Future trends in the casualty application} of ultrasound

Medical provision will continue to evolve. Changes both in the pattern of health care referrals and the technology of medical imaging will impact on ultrasound in casualty.

Ultrasound has taken great strides forwards in technical quality. First the quality of the two dimensional grey scale image has advanced and will continue to advance. By comparison with machines even five years old this makes imaging technically easier.

However the technical capabilities of the machines beyond the generation of a simple two dimensional screen also expand at a prodigious pace. Machines costing up to $£ 200000$ have a litter of advanced capabilities (see table 1). There is a point to be made here in terms of the training that surgical papers have laid down as appropriate to master the technicalities of ultrasound. It has been demonstrated that the basics can be safely mastered and it should be 
appreciated that that is the remit of this mandate. It will take increasing specialisation to keep abreast of all the technical capabilities of current and future generations of machines.

A branch point must realistically be set. Logic is against having a large and highly sophisticated machine in casualty. If the machine is not mobile and the patient has to move to the machine then a large part of the logic of basing the machine in casualty is lost. The larger machines do have limited mobility but the risk in doing so is proportionately greater. Multiple users will make the discipline required to keep the machine in optimum condition harder.

The logic may lie at the opposite extreme as miniaturisation advances at the other end of the technical range. Mobile machines are now very small and light and unlike their recent predecessors have good quality two dimensional imaging capabilities. There is an inherent acceptance that in following the path to "light and simple" there will be imaging questions that will need to be addressed to a central imaging department. It is then a question of predefining which questions can be readily addressed.

Emergency medicine will continue to face change:

(1) A changing pattern of inner city presentation to casualty rather than to general practice continues. Arranging an inpatient diagnostic test to avoid patient admission would rightly be outdated if an imaging question could be answered at presentation. To cope with subacute general practitioner (GP) type presentations that have no specific requirement to be imaged immediately may, however, be an unrealistic pressure on the imaging facility in casualty. The expanding outreach of the GP service may mean that some of these patients will appropriately be imaged in the community.

(2) With the number of casualty attendances continuing to rise cost pressure per patient attendance will continue to tighten. Pressure to provide a same day turn round service with tight focus on costing per finished consultant episode is a strong pressure to find routes to answer imaging questions promptly. As discussed above, a clear distinction should be drawn between providing a rapid diagnosis and necessarily shortening hospital stay.

\section{Conclusion}

As the NHS passes its 50th anniversary change remains ever present. Important new funding may offer the prospect of new investment, pos- sibly hastening the introduction of imaging equipment to casualties. There can be no better way than for this introduction to be managed in close cooperation between radiology and casualty departments both in terms of training and protocols for imaging. Ultrasound that is protocol driven within specific and limited goals will provide fast answers to questions where time is of the essence.

It is likely to remain inappropriate to answer subtle or specialist questions in casualty. Neither the equipment, the time, nor the degree of specialisation are likely nor appropriately available in casualty. The probability that tests will be repeated once under specialist management is high. However the introduction of limited bedside ultrasonography by emergency physicians has the potential to improve diagnostic accuracy and efficiency, increase the quality of care, and prove to be a cost effective aid to the practice of emergency medicine.

Conflict of interest: none.

Funding: none.

1 Ma OJ, Mateer JR, Scalea TM, et al. Trauma ultrasound examination versus chest radiography in the detection of hemothorax. Ann Emerg Med 1997;29:312-16.

2 Laing FC. Ultrasonography of the acute abdomen. Radiol Clin North Am 1992;30:389-404.

3 Cogo A, Lensing A, Koopman M, et al. Compression ultrasonography for diagnostic management of patients with suspected deep vein thrombosis: prospective cohort study. BMF 1998;316:17-20.

4 Shih CHY, Mateer J. Effect of emergency physicianperformed pelvic sonography on length of stay in the emergency department. Ann Emerg Med 1997;29:348-52.

5 Durham B, Lane B, Burbridge L, et al. Pelvic ultrasound performed by emergency physicians for the detection of ectopic pregnancy in complicated first-trimester pregnanectopic pregnancy in complicated first
cies. Ann Emerg Med 1997;29:338-47.

6 Mateer JR, Valley VT, Aiman EJ, et al. Outcome analysis of a protocol including bedside endovaginal sonography in patients at risk for ectopic pregnancy. Ann Emerg Med 1996;27:283-9.

7 Phillips CD. Emergent radiologic evaluation of the gunshot wound victim. Radiol Clin North Am 1992;30:307-24.

8 McKenney K, Nunez D, McKenney M, et al. Sonography as the primary screening technique for blunt abdominal trauma. $A f R$ 1998;170:970-84.

9 Rozycki GS. Abdominal ultrasonography in trauma. Surg Clin North Am 1995;75:175-91.

10 Sarkisian A, Khondkarian R, Amirbekian N, et al. Sonographic screening of the mass casualties for abdominal and renal injuries following the 1988 Armenian earthquake. $\mathcal{f}$ Trauma 1991;31:247-50.

11 Buzzas G, Kern S, Smith S, et al. A comparison of sonographic examinations for trauma performed by surgeons and radiologists. $\mathcal{F}$ Trauma 1998;44:604-8.

12 Mateer J, Plummer D, Heller M, et al. Model curriculum for physician training in emergency ultrasonography. Ann Emerg Med 1994;23:95-102.

13 Ali J, Rozycki GS, Campbell JP, et al. Trauma ultrasound workshop improves physician detection of peritoneal and pericardial fluid. F Surg Res 1996;63:275-9.

14 McKenney M, McKenney $\mathrm{K}$, Compton $\mathrm{R}$, et al. Can surgeons evaluate emergency ultrasound scans for blunt abdominal trauma? F Trauma 1998;44:649-53.

15 Thomas B, Falcone RE, Vasquez D, et al. Ultrasound evaluation of blunt abdominal trauma: program implementation, initial experience and learning curve. $\mathcal{F}$ Trauma 1997; 42:384.

16 Board of Faculty of Clinical Radiology. Guidance for training of medical non-radiologists. London: Royal College of Radiologists, March 1997. 